PROCEEDINGS OF THE

AMERICAN MATHEMATICAL SOCIETY

Volume 133, Number 11, Pages 3437-3440

S 0002-9939(05)07936-0

Article electronically published on May 9, 2005

\title{
A GENERALIZED KAKUTANI-KODAIRA THEOREM
}

\author{
ZHIGUO HU
}

(Communicated by N. Tomczak-Jaegermann)

\begin{abstract}
In this note, we present a generalized Kakutani-Kodaira theorem for all locally compact groups.
\end{abstract}

\section{INTRODUCTION}

Let $G$ be a locally compact group with identity $e$. The well-known classical Kakutani-Kodaira Theorem says that if $G$ is $\sigma$-compact, then, for any sequence $\left\{U_{n}\right\}_{n=1}^{\infty}$ of neighborhoods of $e$, there exists a compact normal subgroup $N$ of $G$ such that $N \subseteq \bigcap_{n=1}^{\infty} U_{n}$ and $G / N$ is metrizable (cf. Kakutani-Kodaira [9]; see also, e.g., Hewitt-Ross [5] Theorem 8.7], Greenleaf-Moskowitz [3, Theorem A.9] and Lau-Losert [10, Remark 14(b)] for a different flavor of proofs). The significance of the Kakutani-Kodaira Theorem has been well demonstrated through its broad applications in harmonic analysis since its introduction in the early 40's. The only limitation of this theorem seems to be the restriction on the $\sigma$-compactness of $G$. However, this limitation is mitigated to a certain extent by the fact that every (non- $\sigma$-compact) locally compact group $G$ contains a $\sigma$-compact open subgroup $G_{0}$. In practice, the Kakutani-Kodaira Theorem is often applied to such $G_{0}$. But in general the resulting compact normal subgroup $N$ of $G_{0}$ may not necessarily be normal in $G$, even though the quotient space $G / N$ of left cosets of $N$ remains metrizable.

In this note, we shall present a generalized Kakutani-Kodaira theorem that applies to all locally compact groups (Theorem 2). The compact subgroup $N$ obtained through this generalized version is assured to be normal in $G$. At first glance, we seem to have to yield the metrizability of $G / N$. However, the degree of the (non)metrizablity of $G / N$ (or the local weight of $G / N$ ) is completely controlled by the compact covering number of $G$, which is exactly the essence of the original Kakotani-Kodaira Theorem.

The formulation of our generalized Kautani-Kodaira theorem stems from the author's study on the dual relation between $L_{\infty}(G)$ and $V N(G)$ as Kac algebras and on how the structure of $L_{\infty}(G), V N(G)$ and the structure of the underlying group $G$ reflect each other (see Hu 8] for more information). Here, $L_{\infty}(G)$ is the abelian von Neumann algebra of multiplication operators on the Hilbert space $L_{2}(G)$, and

Received by the editors November 27, 2003 and, in revised form, June 22, 2004.

2000 Mathematics Subject Classification. Primary 22D05.

Key words and phrases. Locally compact groups, cardinal invariants of locally compact groups, the Kakutani-Kodaira Theorem.

This research was supported by an NSERC grant.

(C)2005 American Mathematical Society Reverts to public domain 28 years from publication 
$V N(G)$ is the von Neumann algebra generated by the left regular representation of $G$. It is well known that both $L_{\infty}(G)$ and $V N(G)$ are Kac algebras which are dual to each other, and together they generate the von Neumann algebra $\mathcal{B}\left(L_{2}(G)\right)$ of all bounded linear operators on $L_{2}(G)$. See Enock-Schwartz [2] for a comprehensive account of the theory of Kac algebras.

\section{Preliminaries}

For any topological space $X$, let $w(X)$ denote the weight of $X$, i.e., the least cardinality of an open basis for $X$. Let $\Omega$ be an infinite locally compact Hausdorff space and let $\Omega_{\infty}$ denote the one-point compactification of $\Omega$. Then $\Omega_{\infty}$ is a compact Hausdorff space and $\Omega$ is open in $\Omega_{\infty}$. Obviously, $\aleph_{0} \leq w(\Omega) \leq w\left(\Omega_{\infty}\right)$. On the other hand, let $\mathcal{E}$ be an open basis for $\Omega$ such that $|\mathcal{E}|=w(\Omega)$. We may assume that each $E \in \mathcal{E}$ is relatively compact in $\Omega$ and $\mathcal{E}$ is closed under finite unions. It is readily seen that $\mathcal{E} \cup\left\{\Omega_{\infty}-c l_{\Omega}(E): E \in \mathcal{E}\right\}$ is an open basis for $\Omega_{\infty}$ and hence $w\left(\Omega_{\infty}\right) \leq|\mathcal{E}|=w(\Omega)$. So, we always have $w\left(\Omega_{\infty}\right)=w(\Omega)$. By a minor modification of the proof of Smirnov Theorem, one can show that dense $\left(C_{0}(\Omega)\right)=w\left(\Omega_{\infty}\right)$ (cf. Hofmann-Morris [6, page 768]), where $C_{0}(\Omega)$ denotes the $C^{*}$-algebra of complexvalued continuous functions on $\Omega$ vanishing at infinity, and $\operatorname{dense}\left(C_{0}(\Omega)\right)$ is the norm density character of $C_{0}(\Omega)$. Therefore, we in fact have

$(\dagger) \quad$ dense $\left(C_{0}(\Omega)\right)=w(\Omega)$ for all infinite locally compact Hausdorff spaces $\Omega$.

In [10, Remark 14(b)], Lau-Losert provided a very elegant and short proof of the classical Kakutani-Kodaira Theorem. Their proof is based on the following result, obtained in the same paper, on the structure of the commutative $C^{*}$-algebra $C_{0}(G)$.

Lemma 1 ([10, Lemma 12]). Let $G$ be a locally compact group. Then $\mathcal{A}$ is a nonzero left translation invariant $C^{*}$-subalgebra of $C_{0}(G)$ if and only if there exists a unique compact subgroup $N_{\mathcal{A}}$ of $G$ such that

$$
\mathcal{A}=\left\{f \in C_{0}(G): f_{t}=f \text { for all } t \in N_{\mathcal{A}}\right\},
$$

where $f_{t}$ denotes the right translate of $f$ by $t$. Furthermore, $\mathcal{A}$ is translation invariant if and only if $N_{\mathcal{A}}$ is normal in $G$.

\section{A generalized Kakutani-Kodaira theorem}

For a locally compact group $G$, we will use $\kappa(G)$ to denote the compact covering number of $G$, and $\chi(G)$ to denote the local weight of $G$, i.e., the least cardinality of a neighborhood basis at the identity $e$ of $G$. With the above preparation, we are now ready to prove the following generalized version of the Kakutani-Kodaira Theorem for all locally compact groups $G$, which clearly includes the classical KakutaniKodaira Theorem as a special case (the case when $\kappa(G) \leq \aleph_{0}$ ). The proof is made amazingly brief by applying Lemma 1 and equality $(\dagger)$ and adopting a similar argument as used in Lau-Losert [10, Remark 14(b)] (thanks to Professor Anthony T. Lau for bringing [10, Remark 14(b)] to our attention).

Theorem 2. Let $G$ be a locally compact group. Then, for any family $\left\{U_{\alpha}\right\}_{\alpha \in \Lambda}$ of neighborhoods of e with $|\Lambda| \leq \kappa(G) \cdot \aleph_{0}$, there exists a compact normal subgroup $N$ of $G$ such that $N \subseteq \bigcap_{\alpha \in \Lambda} U_{\alpha}$ and $\chi(G / N) \leq \kappa(G) \cdot \aleph_{0}$. 
Proof. First, let $U$ be a neighborhood of $e$. Choose a real-valued continuous function $f$ on $G$ with compact support such that $\operatorname{supp} f \subseteq U$ and $f(e)=1$. Let $\mathcal{A}$ be the translation invariant $C^{*}$-subalgebra of $C_{0}(G)$ generated by $f$.

Claim 1: $\operatorname{dense}(\mathcal{A}) \leq \kappa(G) \cdot \aleph_{0}$. Let $n$ be any fixed positive integer. For each $x \in G$, since $f$ is a continuous function on $G$ with compact support, there exists a neighborhood $U_{n}(x)$ of $x$ such that

$$
\left\|{ }_{y} f-{ }_{x} f\right\|<\frac{1}{n} \text { and }\left\|f_{y}-f_{x}\right\|<\frac{1}{n} \text { for all } y \in U_{n}(x),
$$

where ${ }_{y} f$ denotes the left translate of $f$ by $y$. So, there exists a subset $E_{n}$ of $G$ such that $\left|E_{n}\right| \leq \kappa(G) \cdot \aleph_{0}$ and $G=\bigcup_{x \in E_{n}} U_{n}(x)$. We may assume that $e \in E_{n}$ for all $n$. Let

$$
E=\bigcup_{n=1}^{\infty} E_{n} \text { and } \mathcal{D}=\left\{{ }_{x} f_{y}: x, y \in E\right\} .
$$

Then $|E| \leq \kappa(G) \cdot \aleph_{0}$ and so $|\mathcal{D}| \leq \kappa(G) \cdot \aleph_{0}$. Let $\mathcal{Q}_{c}$ be the set of "rational" complex numbers, i.e., $\mathcal{Q}_{c}=\{a+i b: a$ and $b$ are rational numbers $\}$. Let $\mathcal{B}$ denote the set of functions of the form $\sum_{m=1}^{n} c_{m} h_{m}$, where $c_{m} \in \mathcal{Q}_{c}$ and $h_{m}$ is a finite product of elements of $\mathcal{D}$. Then $|\mathcal{B}| \leq|\mathcal{D}| \cdot \aleph_{0} \leq \kappa(G) \cdot \aleph_{0}$. It is readily seen that $\overline{\mathcal{B}}\|\cdot\|$ is the translation invariant $C^{*}$-subalgebra of $C_{0}(G)$ generated by $f$, i.e., $\mathcal{A}=\overline{\mathcal{B}}^{\|\cdot\|}$. It follows that

$$
\operatorname{dense}(\mathcal{A}) \leq|\mathcal{B}| \leq \kappa(G) \cdot \aleph_{0} .
$$

Therefore, Claim 1 is proved.

Owing to Lemma 1, there must exist a compact normal subgroup $N$ of $G$ such that $\mathcal{A}=C_{0}(G / N)$.

Claim 2: $\chi(G / N) \leq \kappa(G) \cdot \aleph_{0}$ and $N \subseteq U$. Obviously, $\chi(G / N) \leq \kappa(G) \cdot \aleph_{0}$ if $G / N$ is finite. Assume that $G / N$ is infinite. Combining Claim 1 with equality (†), we have

$$
\chi(G / N) \leq w(G / N)=\operatorname{dense}\left(C_{0}(G / N)\right)=\operatorname{dense}(\mathcal{A}) \leq \kappa(G) \cdot \aleph_{0} .
$$

Furthermore, for all $t \in N$, since $f_{t}=f, f(t)=f(e)=1$. In particular, we have $N \subseteq \operatorname{supp} f \subseteq U$. Therefore, Claim 2 holds.

In general, let $\left\{U_{\alpha}\right\}_{\alpha \in \Lambda}$ be an arbitrary family of neighborhoods of $e$ such that $|\Lambda| \leq \kappa(G) \cdot \aleph_{0}$. Let us consider the functions $f_{\alpha}$ for all $U_{\alpha}$ as we choose $f$ for $U$. Let $\mathcal{A}$ be the translation invariant $C^{*}$-subalgebra of $C_{0}(G)$ generated by $\left\{f_{\alpha}\right\}_{\alpha \in \Lambda}$. Then, by Lemma 1 again, $\mathcal{A}=C_{0}(G / N)$ for some compact normal subgroup $N$ of $G$. Since $|\Lambda| \leq \kappa(G) \cdot \aleph_{0}$, it is readily seen from the proof of Claim 1 that dense $(\mathcal{A}) \leq \kappa(G) \cdot \aleph_{0}$. It follows that

$$
\chi(G / N) \leq w(G / N) \leq \operatorname{dense}\left(C_{0}(G / N)\right)=\operatorname{dense}(\mathcal{A}) \leq \kappa(G) \cdot \aleph_{0} .
$$

Moreover, for each $\alpha \in \Lambda$, since $f_{\alpha} \in C_{0}(G / N)$ and $f_{\alpha}(t)=f_{\alpha}(e)=1$ for all $t \in N$, we have $N \subseteq \operatorname{supp} f_{\alpha} \subseteq U_{\alpha}$. Therefore, $N \subseteq \bigcap_{\alpha \in \Lambda} U_{\alpha}$. The proof is complete.

Remark 3 . As a special case when $G$ is a $\sigma$-compact locally compact group, Theorem 2 naturally reduces to the classical Kakutani-Kodaira Theorem. This is because $G$ is $\sigma$-compact if and only if $\kappa(G) \leq \aleph_{0}$ and the quotient group $G / N$ is metrizable if and only if $\chi(G / N) \leq \aleph_{0}$ (cf. [5, Theorem 8.5]).

Remark 4 . Let $\kappa$ be any cardinal number. A subset $E$ of $G$ is called $\kappa$-open in $G$ if $E$ is an intersection of no more than $\kappa$ open subsets of $G$. Let $\gamma(E)$ denote the 
least cardinal number $\kappa$ such that $E$ is $\kappa$-open in $G$. Adopting the argument used in Hewitt-Ross [4, (28.69(a))], we can obtain the following equality:

$$
\chi(G / N)=\gamma(N) \text {. }
$$

Furthermore, combining $(\ddagger)$ with $\mathrm{Hu}$ [7, Lemma 4.2] (which implies that $\chi(G) \leq$ $\gamma(N) \cdot \chi(N))$ and noting that $\chi(N) \leq \chi(G)$ and $\chi(G / N) \leq \chi(G)$, we conclude that

$$
\chi(G)=\chi(G / N) \cdot \chi(N) .
$$

Obviously, Theorem 2 is trivial if $\chi(G) \leq \kappa(G) \cdot \aleph_{0}$. The equality (\#) tells us that if $\kappa(G) \cdot \aleph_{0}<\chi(G)$, then the non-trivial compact normal subgroup $N$ of $G$ obtained in Theorem 2 satisfies $\chi(N)=\chi(G)$. Hence, in this case, we also have $w(N)=w(G)$ and $|N|=|G|$ (cf. Comfort 1, Theorem 3.5(iii) and Theorem 3.12 (iii)]). On the other hand, since $\kappa(G / N)=\kappa(G)$ (cf. Hewitt-Ross [5. (5.24(a))]) and $\chi(G / N) \leq \kappa(G) \cdot \aleph_{0}=\kappa(G / N) \cdot \aleph_{0}$, the abelian von Neumann algebra $L_{\infty}(G / N)$ is a so-called maximally decomposable sub-Kac algebra of $L_{\infty}(G)$ (cf. Hu [8]).

The author thanks the referee for the valuable suggestions, in particular, for suggesting the simplified proof of Theorem 2 presented here.

\section{REFERENCES}

1. W. W. Comfort, Topological groups, Chapter 24 in: K. Kunen and J. E. Vaughan (eds.), Handbook of Set-Theoretic Topology, North-Holland, New York, 1984, 1143-1263. MR0776643 (86g:22001)

2. M. Enock and J.-M. Schwartz, Kac Algebras and Duality of Locally Compact Groups, Springer-Verlag, New York, 1992. MR 1215933 (94e:46001)

3. F. P. Greenleaf and M. Moskowitz, Cyclic vectors for representations associated with positive definite measures: nonseparable groups, Pacific J. Math. 45 (1973), 165-186. MR0349896 (50:2389)

4. E. Hewitt and K. A. Ross, Abstract Harmonic Analysis II, Springer-Verlag, New York, 1970. MR0262773 (41:7378)

5. E. Hewitt and K. A. Ross, Abstract Harmonic Analysis I, Springer-Verlag, New York, 1979. MR0551496(81k:43001)

6. K. H. Hofmann and S. A. Morris, The Structure of Compact Groups: A Primer for the Student - A Handbook for the Expert, de Gruyter Studies in Math. 25, Walter de Gruyter and Co., Berlin, 1998. MR1646190 (99k:22001)

7. Z. $\mathrm{Hu}$, On the set of topologically invariant means on the von Neumann algebra $V N(G)$, Illinois J. Math. 39 (1995), 463-490. MR1339838 (96e:22012)

8. Z. Hu, Maximally decomposable von Neumann algebras on locally compact groups and duality, Preprint, 2004.

9. S. Kakutani and K. Kodaira, Über das Haarsche Mass in der lokal bikompakten Gruppe, Proc. Imp. Acad. Tokyo 20 (1944), 444-450. MR0014401(7:279d)

10. A. T. Lau and V. Losert, Complementation of certain subspaces of $L_{\infty}(G)$ of a locally compact group, Pacific J. Math. 141 (1990), 295-310. MF1035445 (91g:43008)

Department of Mathematics and Statistics, University of Windsor, Windsor, OnTARIO, CANADA N9B 3P4

E-mail address: zhiguohu@uwindsor.ca 